\title{
Article \\ Feasibility of the Quantitative Assessment Method for CT Quality Control in Phantom Image Evaluation
}

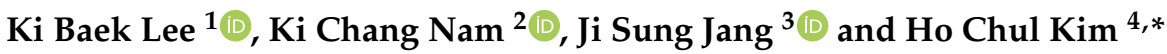 \\ 1 Biomedical Engineering Research Center, Asan Institute for Life Sciences, Asan Medical Center, \\ 88 Olympic-ro 43-gil, Songpa-gu, Seoul 05505, Korea; beall34@amc.seoul.kr \\ 2 Department of Medical Engineering, Dongguk University College of Medicine, 32 Dongguk-ro, Goyang-si, \\ Gyeonggi-do 10326, Korea; chadol@dongguk.ac.kr \\ 3 Department of Radiology and Research Institute of Radiology, University of Ulsan College of Medicine, \\ Asan Medical Center, Seoul 05505, Korea; jsjang@amc.seoul.kr \\ 4 Department of Radiological Science, Eulji University, 553 Sanseong-daero, Sujeong-gu, Seongnam-si, \\ Gyeonggi-do 13135, Korea \\ * Correspondence: tiger1005@eulji.ac.kr; Tel.: +82-031-740-7412
}

check for updates

Citation: Lee, K.B.; Nam, K.C.; Jang, J.S.; Kim, H.C. Feasibility of the Quantitative Assessment Method for CT Quality Control in Phantom Image Evaluation. Appl. Sci. 2021, 11, 3570. https://doi.org/10.3390/app 11083570

Academic Editor: Qi-Huang Zheng

Received: 9 March 2021

Accepted: 12 April 2021

Published: 16 April 2021

Publisher's Note: MDPI stays neutral with regard to jurisdictional claims in published maps and institutional affiliations.

Copyright: (c) 2021 by the authors. Licensee MDPI, Basel, Switzerland. This article is an open access article distributed under the terms and conditions of the Creative Commons Attribution (CC BY) license (https:// creativecommons.org/licenses/by/ $4.0 /)$.

\begin{abstract}
Computed tomography (CT) quality control (QC) is regularly performed with standard phantoms, to bar faulty equipment from medical use. Its accuracy may be improved by replacing qualitative methods based on good visual distinction with pixel value-based quantitative methods. We hypothesized that statistical texture analysis (TA) that covers the entire phantom image would be a more appropriate tool. Therefore, our study devised a novel QC method based on the TA for contrast resolution (CR) and spatial resolution (SR) and proposed new, quantitative CT QC criteria. TA of CR and SR images on an American Association of Physicists in Medicine (AAPM) CT Performance Phantom were performed with nine CT scanner models. Six texture descriptors derived from first-order statistics of grayscale image histograms were analyzed. Principal component analysis was used to reveal descriptors with high utility. For CR evaluation, contrast and softness were the most accurate descriptors. For SR evaluation, contrast, softness, and skewness were the most useful descriptors. We propose the following ranges: contrast for CR, $29.5 \pm 15 \%$, for SR, $29 \pm 10 \%$; softness for $C R,<0.015$, for $S R,<0.014$; and skewness for $S R,>-1.85$. Our novel TA method may improve the assessment of CR and SR of AAPM phantom images.
\end{abstract}

Keywords: computed tomography; quality control; contrast resolution; spatial resolution; texture analysis

\section{Introduction}

Computed tomography (CT) is a computerized imaging technique in which many X-ray measurements of a body are made, with an X-ray tube usually rotating around the patient during the CT scan [1,2]. These signals are computationally processed and, consequently, cross-sectional images of the body are reconstructed. The utilization of CT for image-based diagnosis has increased steeply in the medical field [3,4]. Additionally, in the past ten years, state of-the-art CT detectors and sources and several techniques, such as automatic exposure control and iterative reconstruction, have been launched; these can provide a higher image quality by using a lower radiation dose [5-10]. However, despite the reduced radiation exposure per single $\mathrm{CT}$ examination, the total number of $\mathrm{CT}$ examinations performed has increased consistently [11,12]. Therefore, it is imperative that CT scanners are properly managed and quality control (QC) is accurately performed [13-16].

QC related to radiation dose and image quality for $\mathrm{CT}$ is universally composed of quantitative assessments with a standard phantom [13-16]. In particular, the decreases in image quality of CT scanners need to be managed quantitatively because a relatively high radiation dose may be used to overcome the image quality during CT examination. Although the standard phantom evaluation exists, this is often included in the quantitative evaluation, 
especially for contrast resolution (CR) and spatial resolution (SR) assessments [14-16]. For the $C R$ and the $S R$ evaluation, radiologists sometimes perform visual evaluations of these images (e.g., holes that are visible as distinct circles), instead of making use of quantitative cut-off values. However, quantitative tools are considered more effective for evaluating phantom images [17-19]. There are several types of standard phantoms with similarities in composition such as the American Association of Physicists in Medicine (AAPM) CT Performance Phantom, and the American College of Radiology (ACR) phantom [14,17,18]. As a result, measurement of the contrast-to-noise ratio $(\mathrm{CNR})$ as an objective tool has been conducted in recent years to evaluate the CR with the ACR phantom in the United States, which overcomes the limitations of qualitative evaluation [17]. However, as the CNR is usually focused on specific regions of interest (ROIs) and does not encompass the entire CT image, there is a risk of sampling error [20]. In fact, a specific location with a small size can be problematic as it represents the quality of the overall image. Therefore, computer-aided analysis, such as texture analysis (TA), that covers the entire ROI was considered as a more appropriate tool than the value of the CNR, which varies by measured location, because it has a lower standard error.

Such tools have already been investigated, making use of the TA, which may be able to distinguish between diseases with objective values [21-24]. There are five types of TA methods: structural, statistical, local binary patterns, model-based, or transform-based [25,26]. Among these, the statistical method has been utilized as the most common approach for CT TA or digital image-based diagnosis $[25,27]$. Since the specific characteristics of the tumor can be distinguished through TA [21-24], we hypothesized that statistical TA may also be useful for classification of standard phantom images during QC of CT scanners. CR and SR represent whether the pixel value in the ROI can be identified in a situation where the difference of signal between the ROI and the surrounding area is small or large. The rationale for this study was whether it would be possible to quantify the distinction for CT QC, similarly to the distinction of tumors through TA mentioned above. Therefore, the purpose of our study was to perform the TA based on the statistical method for CR and SR assessment and to evaluate its feasibility as a quantitative component of CT QC.

\section{Materials and Methods}

\subsection{Equipment}

An AAPM CT Performance Phantom (Model 76-410; Fluke Corporation, Everett, WA, USA) was used for all experiments. It is a cylinder made of acrylic and consists of five different assessment blocks (Figure 1a). We utilized multi-detector CT scanners made by various manufacturers, including widely used and state-of-the-art equipment in three tertiary hospitals. The scanner, rows of detectors, and manufacturer's information were as follows: SOMATOM Definition AS (32), AS+ (64), and Edge (64) (Siemens Healthineers, Erlangen, Germany); Lightspeed VCT XTe (64) and Discovery CT750 HD (64) (GE Healthcare, Chicago, IL, USA); Phillips Brilliance CT (64) and IQon Spectral CT (64) (Philips Healthcare, Amsterdam, Netherlands); and Aquilion CX (64) and GENESIS (160) (Canon Medical Systems Corporation, Otawara, Japan). The SOMATOM Edge differs from the AS+ only in its integrated circuit detector, which provides a higher image quality [6]. Similarly, the Discovery CT750 HD contains a gemstone clarity detector, which generates higherresolution images than those of the Lightspeed VCT XTe [7]. The former is state-of-the-art equipment, and the latter is a widely used scanner. 


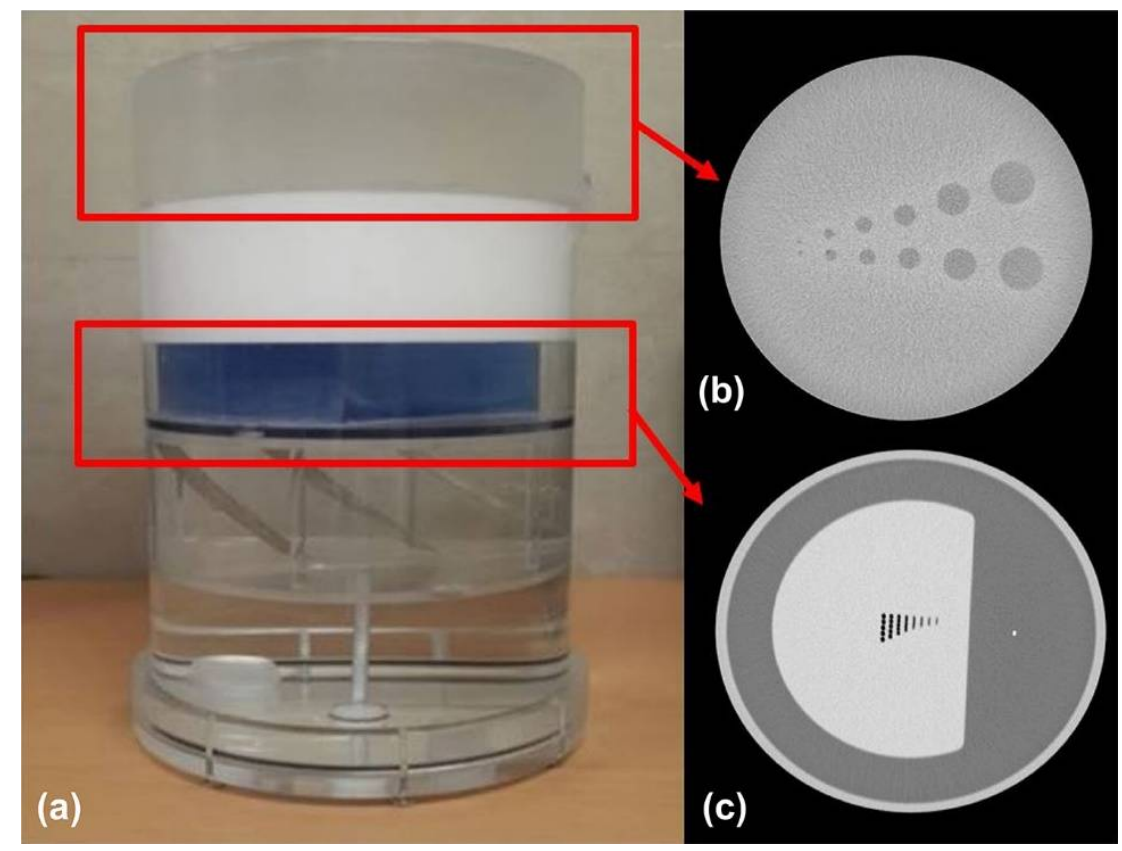

Figure 1. The American Association of Physicists in Medicine (AAPM) phantom. (a) The AAPM CT Performance Phantom consists of five blocks with different functions, from top to bottom: contrast resolution (CR), linearity, spatial resolution (SR), slice thickness, and various measurements; (b) the CR block; (c) the SR block.

\subsection{Image Acquisition Parameters}

For CT QC, image acquisition parameters were equal for all scanners, except for the tube current (in $\mathrm{mA}$ ). The recommended tube current is $250 \mathrm{~mA}$ for AAPM CT Performance Phantom evaluation $[13,14]$. However, the CT radiation dose, measured as the CT dose index volume (CTDIvol), is not the same among the various CT scanners when the tube current is fixed at $250 \mathrm{~mA}$. As a result, we adopted a radiation dose-based protocol (CTDIvol: $20 \mathrm{mGy}$ ) for all scanners (i.e., a tube current corresponding to a CTDIvol of $20 \mathrm{mGy}$ was selected). However, CT scanners that cannot be set to exactly $20 \mathrm{mGy}$ were set to the nearest value) (Table 1), similar to that of a previous study [28]. The other parameters were as follows: tube peak kilovoltage $(\mathrm{kVp})=120 \mathrm{kV}$, rotation time $=1 \mathrm{~s}$, reconstruction method: filtered back projection, convolution kernels = Siemens, B30; GE, standard; Philips, B; Canon, FC13, display field-of-view $=25 \mathrm{~cm}$, and scan mode $=$ sequential scan of 10-mm slice thickness. All raw data in DICOM format was acquired by one author (K.B.L. with 10 years of experience in $\mathrm{QC}$ of $\mathrm{CT}$ ).

Table 1. Tube current values of CT scanners to achieve a CTDIvol of $\pm 20 \mathrm{mGy}$.

\begin{tabular}{|c|c|c|c|c|c|c|c|}
\hline \multirow{2}{*}{ Manufacturer } & \multirow{2}{*}{ Model } & Tube Voltage & Rotation Time & Slice Thickness & Convolution Kernel & Tube Current & $\mathrm{CTDI}_{\mathrm{vo}}$ \\
\hline & & $\mathbf{k V p}$ & $\mathbf{s}$ & $\mathrm{mm}$ & & $\mathrm{mA}$ & mGy \\
\hline \multirow{3}{*}{ Siemens } & Edge & & & & & 274 & 19.95 \\
\hline & $\mathrm{AS}+$ & & & & B30f & 300 & 19.92 \\
\hline & AS & & & & & 300 & 19.92 \\
\hline \multirow[b]{2}{*}{ GE } & CT750 HD & & & & & 225 & 19.85 \\
\hline & VCT XTe & 120 & 1 & 10 & Standard & 235 & 19.9 \\
\hline \multirow{2}{*}{ Philips } & IQon & & & & & 230 & 20 \\
\hline & Brilliance & & & & B & 305 & 20 \\
\hline \multirow[b]{2}{*}{ Canon } & GENESIS & & & & & 350 & 19.9 \\
\hline & Aquilion CX & & & & FC13 & 170 & 20 \\
\hline
\end{tabular}

The specifications of the scanners are as follows: Siemens Healthineers: SOMATOM Definition AS (32), AS+ (64), and Edge (64); GE Healthcare: Lightspeed VCT XTe (64) and 
Discovery CT750 HD (64); Philips Healthcare: Brilliance CT (64) and IQon Spectral CT (64); Canon Medical Systems: Aquilion CX (64) and GENESIS (160). CT, computed tomography; CTDIvol, computed tomography dose index volume; $\mathrm{kVp}$, peak kilovoltage.

\subsection{Acquisition Method of CR Images}

The CR block contains holes of $57.2 \mathrm{~mm}$ in depth and 25.4, 19.1, 12.7, 9.5, 6.4, and $3.2 \mathrm{~mm}$ in diameter, at intervals of $5 \mathrm{~mm}$, arranged in pairs separated by twice the width of the respective holes (Figure 1b). CR-block images were acquired with four different contrast medium concentrations and in eight different positions $(12,1.5,3,4.5,6,7.5,9$, and 10.5 o'clock) with each machine. In particular, the methodology of acquiring images with different directions aimed to examine the validity of the related result values by considering all directions that can be acquired at the time of actual QC. For CR evaluation, the required difference in radiodensity between the holes and the ROIs (the acrylic medium between the holes) was $10 \mathrm{HU}$ (Hounsfield units). However, there are no specific criteria for contrast medium concentration for CR evaluation. Therefore, we utilized four different concentrations to measure the radiodensity, in order to prevent users from exploiting the maximum concentration to achieve the required $C R$. The contrast medium used was $0.612 \mathrm{~g} / \mathrm{mL}$ iopamidol, of which $0.3 \mathrm{~g} / \mathrm{mL}$ was iodine (Pamiray 300; DongKook Pharmaceutical Co. Ltd., Seoul, Korea) and the concentrations used were 0.01\%, (Figure 2a), 0.015\% (Figure 2b), 0.02\% (Figure 2c), and 0.025\% (Figure 2d) of the contrast medium diluted in sterile distilled water.

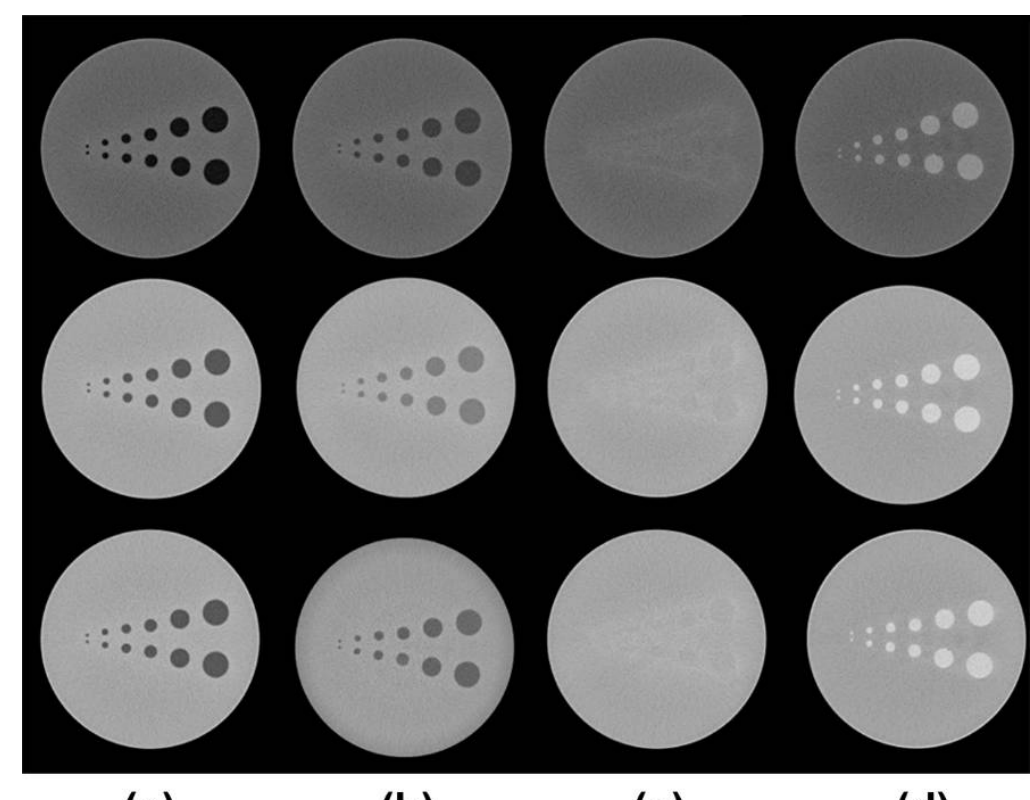

(a)

(b)

(c)

(d)

Figure 2. Images acquired for $C R$ evaluation in the 3 o'clock position. From a to $d$, images were acquired with four different concentrations of contrast medium: $0.01 \%(\mathbf{a}), 0.015 \%(\mathbf{b}), 0.02 \%$ (c), and $0.025 \%$ (d). From top to bottom, images were acquired using the SOMATOM Definition AS, AS+, and Edge CT scanners (Siemens Healthineers).

\subsection{Acquisition Method of SR Images}

SR images were acquired in four different positions (12, 3, 6, and 9 o'clock). The rationale of the acquisition of images in various directions is the same as the reason shown in the acquisition method of CR images. The SR block contains five rows with eight holes each, with a diameter of $1.75,1.50,1.25,1.00,0.75,0.60,0.50$, and $0.40 \mathrm{~mm}$, at intervals of $4.3 \mathrm{~mm}$ (Figure 1c). Since the SR evaluation aims to evaluate the sharpness of small objects with high-density differences, inadequate images were acquired and compared with appropriate images for comparison of the sharpness. In order to compare images 
with appropriate (Figure 3a) and distorted SR (Figure 3b), distorted images were acquired in the same four positions by tilting the gantry of the CT scanner 10 degrees backward (Dist_B) or forward (Dist_F). The suspension rate of SR represented deviation $\geq 10 \%$ from the manufacturer's specification [16]. Thus, the angle of 10 degrees was used to distort the image.

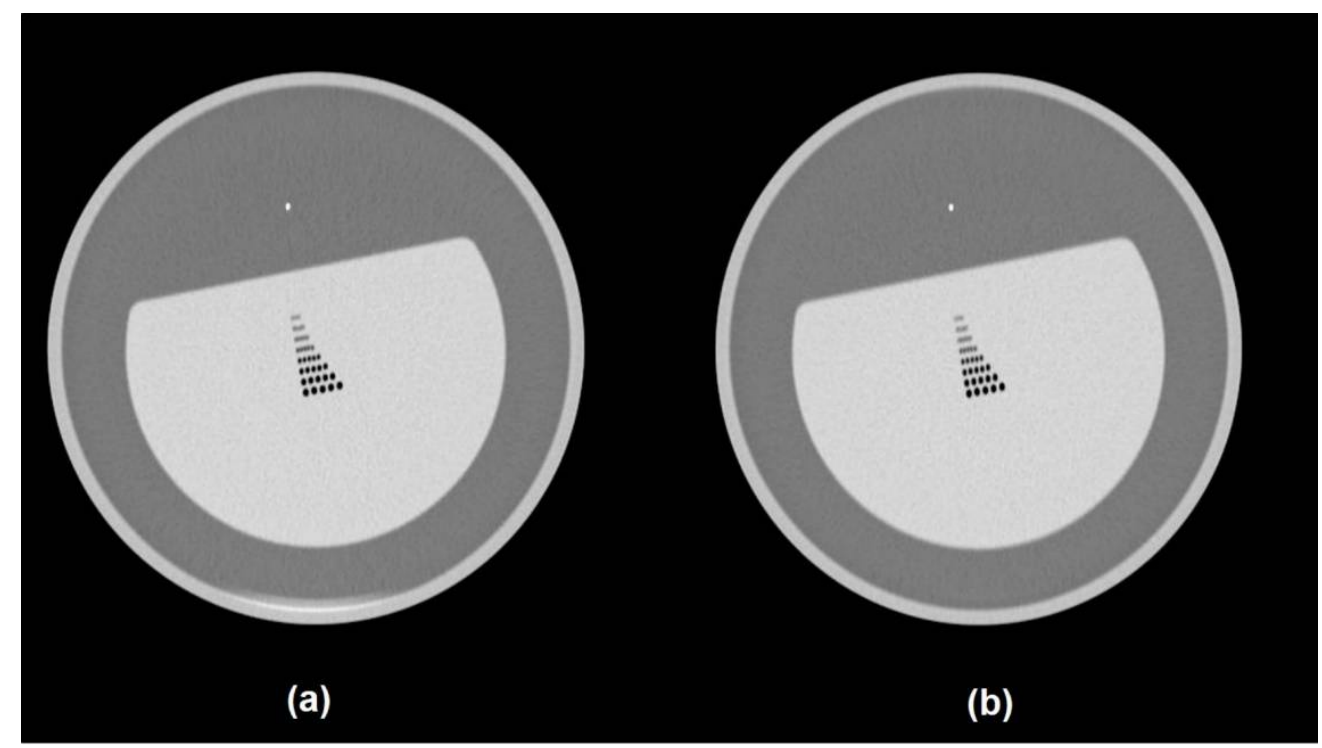

Figure 3. Example images with appropriate and distorted spatial resolution (SR). (a) An image with appropriate SR can be used to visually separate the four largest groups of holes; (b) An image with inappropriate SR, distorted by tilting the gantry of the CT scanner.

\subsection{Quantitative Analysis}

\subsubsection{Texture Analysis (TA)}

Quantitative assessment was performed using an evaluation tool that was developed in the MATLAB/Simulink 7.10.0 platform (R2015a; MathWorks, Natick, MA, USA). A total of six texture descriptors were evaluated, derived from first-order statistics of grayscale image histograms: brightness, contrast, softness, skewness, uniformity, and randomness [29-33]. The specific definition, equation, and characteristics were shown in Table 2.

Table 2. A total of six texture descriptors from first-order statistics of grayscale image histograms.

\begin{tabular}{|c|c|c|c|}
\hline Definition & Equation & Description & Characteristics \\
\hline Brightness & $\sum_{i=0}^{L-1} z_{i} p\left(z_{i}\right)$ & $\begin{array}{l}\text { Mean gray level (pixel value) of the image (equal } \\
\text { to the mean value in the histogram) }\end{array}$ & \\
\hline Contrast & $\sqrt{\sigma^{2}}$ & Variance from the mean value & \\
\hline Softness & $1-1 /\left(1+\sigma^{2}\right)$ & Softness is a relative measure of image brightness & $\begin{array}{l}\text { The softness index closer to } 0 \text { represents an } \\
\text { image with more constant brightness }\end{array}$ \\
\hline Skewness & $\sum_{i=0}^{L-1}\left(z_{i}-m\right)^{3} p\left(z_{i}\right)$ & Degree of asymmetry of the histogram & $\begin{array}{l}\text { If the histogram is symmetrical, the skewness } \\
\text { index is } 0 \text {. If high pixel values are located to the } \\
\text { right or left from the mean value, the skewness } \\
\text { is a positive or negative value }\end{array}$ \\
\hline Uniformity & $-\sum_{i=0}^{L-1} p^{2}\left(z_{i}\right)$ & Similarity of gray levels & $\begin{array}{l}\text { Uniformity is at its highest when light and shade } \\
\text { are distributed uniformly throughout the figure }\end{array}$ \\
\hline Randomness & $\sum_{i=0}^{L-1} p\left(z_{i}\right) \log _{2} p\left(z_{i}\right)$ & Entropy & $\begin{array}{l}\text { Randomness is at its lowest under the } \\
\text { same conditions }\end{array}$ \\
\hline
\end{tabular}

Note. $z$ is a random variable representing brightness, $p(z)$ is a histogram of brightness levels of the ROI, and $L$ is the number of possible brightness levels. 


\subsubsection{Analysis Method of Image CR}

To apply the evaluation tool for $\mathrm{CR}$, the biggest holes in the $\mathrm{CR}$ block must be arrayed on the left side of the image; this is achieved by drawing a line that connects the largest holes, allowing the tool to orient the image (Figure $4 a, b$ ). Thereafter, the ROI is indicated to the tool by placing a $300 \times 200$ px rectangle so that it encompasses all 12 holes (Figure 4c). The algorithm then automatically calculates the six TA descriptors from the ROI image (Figure 4d).

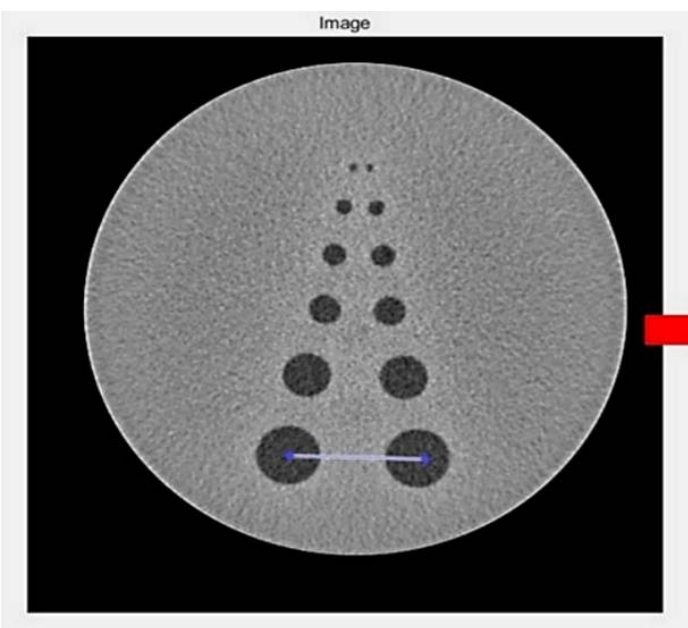

(a)

(c)

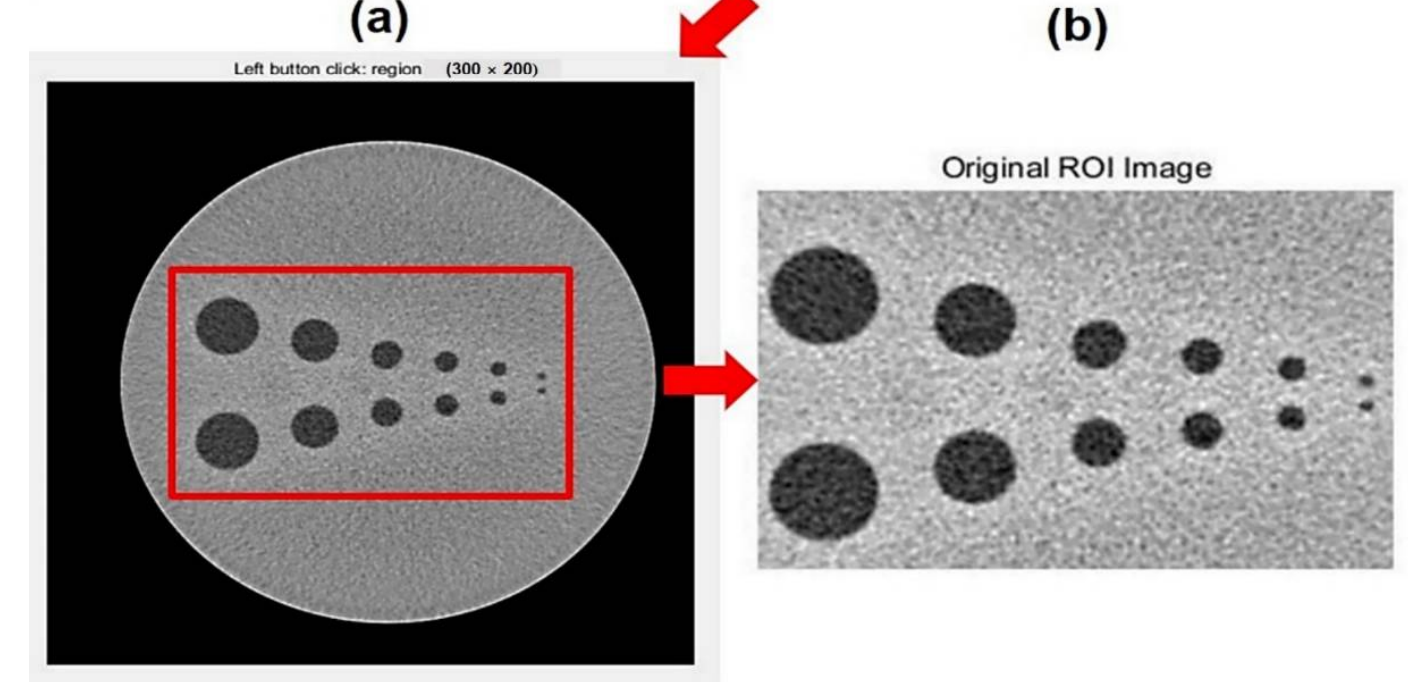

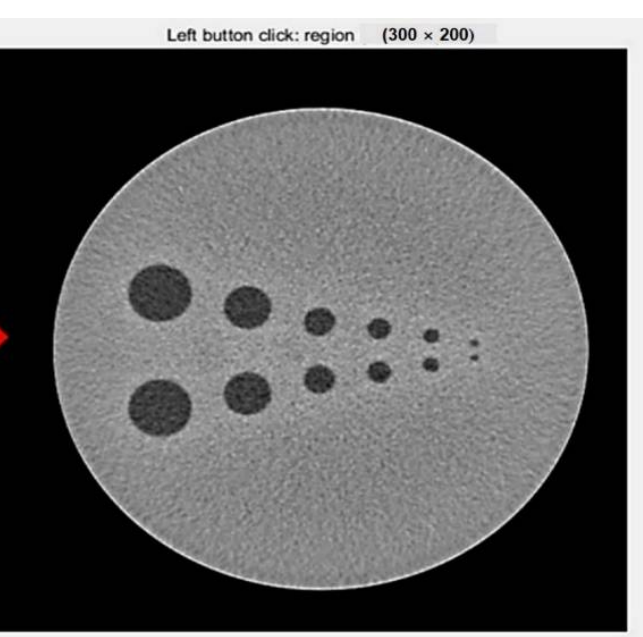

(b)

(d)

Figure 4. Operation of the contrast resolution (CR) quantification tool. (a) The original orientation of the image, with the biggest holes positioned at 6 o'clock. The user draws a line between the two biggest holes. (b) The tool correctly aligns the image. (c) A region of interest (ROI) of $300 \times 200$ px was selected in this image, to encompass all 12 holes. (d) The tool performs texture analysis (TA) on the ROI image.

\subsubsection{Analysis Method of Image SR}

For CR evaluation, the largest holes in the SR block need to be arrayed on the left side of the image. This is a simple step to apply images acquired from various directions to our program. This is achieved by drawing a line over a stainless-steel wire located on the opposite side, which is normally used for modulation transfer function evaluation (Figure 5a). Using this indication, the tool automatically orients the image (Figure 5b). Lastly, the ROI is indicated to the tool by placing an $80 \times 60 \mathrm{px}$ rectangle so that it 
encompasses all 40 holes (Figure 5b). Thereafter, the tool automatically calculates the six

TA descriptors for the ROI (Figure 5c).

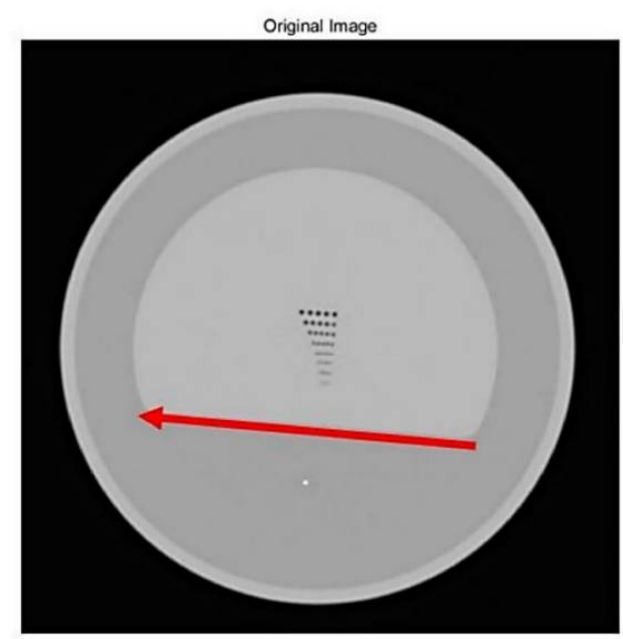

(a)

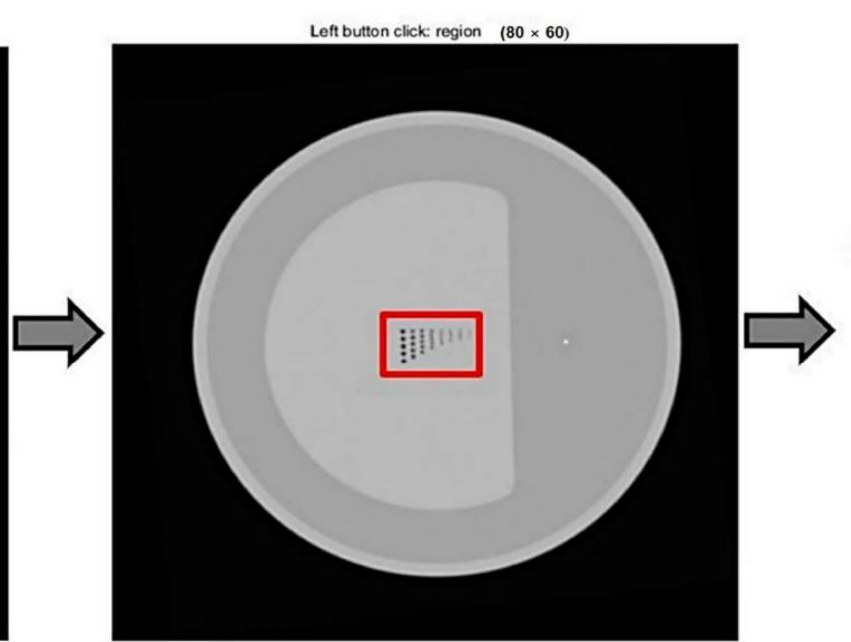

(b)
Original ROI Image

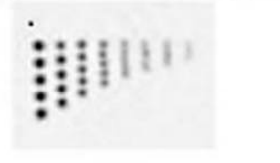

Figure 5. Operation of the spatial resolution (SR) quantification tool. (a) The original orientation of the image with the biggest holes positioned at approximately 12 o'clock. The user draws a line over the stainless steel wire; (b) the tool correctly aligns the image. A region of interest (ROI) of $80 \times 60$ px was selected in this image; (c) the tool performs texture analysis (TA) on the ROI image.

\subsection{Statistical Analysis}

All six TA descriptors for CR and SR (appropriate and distorted images) were measured in each of the different imaging locations and compared in the same descriptors respectively. For $C R$, TA descriptors of $C R$ images yielding the lowest difference in contrast between holes and background were compared within all CT scanners using a one-way analysis of variance (no adjustments were made for multiple comparisons). Afterwards, CR TA descriptors were also compared between different scanner models of the same manufacturers, using either a two-tailed, independent two-sample $t$-test (comparing two models) or a one-way analysis of variance (comparing three models; no adjustments were made for multiple comparisons). SR TA descriptors were compared between appropriate and distorted images using a two-tailed, paired $t$-test, and between different scanner models of the same manufacturers, using either a two-tailed, independent two-sample $t$-test or a one-way analysis of variance.

Principal component analysis was used to determine the texture descriptors with the most utility for CR and SR evaluation. First, Kaiser-Meyer-Olkin and Bartlett's test of sphericity was used to evaluate the case-to-variable ratio. Thereafter, the rotated component matrix was obtained and simplified using varimax rotation [34]. A $p$-value $<0.05$ was considered statistically significant.

\section{Results}

\section{1. $T A$ of $C R$}

There were no statistically significant differences in CR TA descriptors in the eight imaging locations within each CT scanner $(p>0.05)$. The contrast medium concentrations yielding the lowest difference in contrast for all CT scanners were $0.015 \%$ or $0.02 \%$ (i.e., when the difference between holes and background was minimum according to the various scanners, the contrast medium diluted in sterile distilled water was as follows: Siemens; $0.02 \%$ in AS, AS+, and Edge, GE; $0.015 \%$ in CT750 HD, and VCT XTe; Philips; $0.015 \%$ in IQon, and $0.02 \%$ in Brilliance, Canon; $20 \%$ in GENESIS, $15 \%$ in Aquilion CX). Contrast and softness were the only descriptors which had no statistically significant differences of CR images yielding the lowest difference in contrast within all CT scanners $(p>0.05)$. Among 
the measured texture descriptors, all values except for brightness showed results reflecting qualitative image characteristics. The other five factors achieved a Kaiser-Meyer-Olkin value of 0.608 , indicating that principal component analysis was suitable. Based on the rotated component matrix, the contrast (0.871), and softness (0.847) indices were determined as suitable indices to evaluate the CR. Here, mean values of skewness, uniformity, and randomness were statistically significantly different between different scanners from the same manufacturer $(p<0.05)$. As a result, contrast and softness were revealed as appropriate indices to evaluate the $C R$, as the differences in their mean values on the lowest-contrast images between different scanners of the same manufacturer were not statistically significant (Table 3). The overall contrast and softness index values were $29.73 \pm 1.36$ and $0.0135 \pm 0.0012$, respectively.

Table 3. Contrast resolution (CR) texture analysis (TA) for all CT scanners using the lowest-contrast image.

\begin{tabular}{|c|c|c|c|c|c|c|c|c|}
\hline MF & Model & $\mathrm{CC}$ & Brightness $\ddagger$ & Contrast $\ddagger$ & Softness $\ddagger$ & Skewness $\ddagger$ & Uniformity $\ddagger$ & Randomness $\ddagger$ \\
\hline \multirow{4}{*}{ Siemens } & AS & 0.02 & $127.65 \pm 2.02$ & $30.56 \pm 1.60$ & $0.0146 \pm 0.0016$ & $0.0024 \pm 0.0125$ & $0.0533 \pm 0.0018$ & $4.4426 \pm 0.0453$ \\
\hline & AS+ & 0.02 & $125.26 \pm 4.65$ & $30.53 \pm 1.40$ & $0.0141 \pm 0.0013$ & $0.0014 \pm 0.0153$ & $0.0568 \pm 0.0022$ & $4.3486 \pm 0.0588$ \\
\hline & EDGE & 0.02 & $125.06 \pm 5.16$ & $30.58 \pm 1.54$ & $0.0142 \pm 0.0014$ & $0.0022 \pm 0.0076$ & $0.0573 \pm 0.0011$ & $4.3382 \pm 0.0249$ \\
\hline & $p$-value $*$ & & 0.047 & 0.996 & 0.760 & 0.887 & $<0.001$ & $<0.001$ \\
\hline \multirow{3}{*}{ GE } & CT750HD & 0.015 & $159.79 \pm 3.52$ & $28.94 \pm 1.11$ & $0.0127 \pm 0.0010$ & $-0.2475 \pm 0.0344$ & $0.0367 \pm 0.0004$ & $5.0469 \pm 0.0111$ \\
\hline & VCT XTe & 0.015 & $\begin{array}{c}135.61 \pm 2.79 \\
<0.001\end{array}$ & $\begin{array}{c}29.91 \pm 1.01 \\
0.084\end{array}$ & $\begin{array}{c}0.0136 \pm 0.0009 \\
0.084\end{array}$ & $\begin{array}{c}-0.0621 \pm 0.0050 \\
<0.001\end{array}$ & $0.0419 \pm 0.0007$ & $\begin{array}{c}4.8016 \pm 0.0215 \\
<001\end{array}$ \\
\hline & $\begin{array}{l}p \text {-value }{ }^{\dagger} \\
\text { IQon }\end{array}$ & 0.015 & $125.04 \pm 3.01$ & $28.73 \pm 1.58$ & $0.0122 \pm 0.0013$ & $\begin{array}{c}<0.001 \\
-0.0363 \pm 0.0180\end{array}$ & $\begin{array}{c}<0.001 \\
0.0362 \pm 0.0004\end{array}$ & $\begin{array}{c}<0.001 \\
5.0213 \pm 0.0190\end{array}$ \\
\hline \multirow[t]{2}{*}{ Philips } & $\begin{array}{l}\text { Brilliance } \\
p \text {-value }^{\dagger}\end{array}$ & 0.02 & $\begin{array}{l}115.10 \pm 5.44 \\
<0.001\end{array}$ & $\begin{array}{c}30.04 \pm 1.28 \\
0.061\end{array}$ & $\begin{array}{c}0.0145 \pm 0.0016 \\
0.055\end{array}$ & $\begin{array}{c}0.2210 \pm 0.0634 \\
<0.001\end{array}$ & $\begin{array}{c}0.0388 \pm 0.0012 \\
<0.001\end{array}$ & $\begin{array}{c}4.9402 \pm 0.0534 \\
<0.001\end{array}$ \\
\hline & GENESIS & 0.02 & $123.95 \pm 2.69$ & $28.83 \pm 1.05$ & $0.0126 \pm 0.0009$ & $0.0170 \pm 0.0043$ & $0.0425 \pm 0.0002$ & $4.7843 \pm 0.0051$ \\
\hline Canon & $\begin{array}{c}\text { Aquilion CX } \\
p \text {-value }\end{array}$ & 0.015 & $\begin{array}{c}122.20 \pm 2.98 \\
0.271\end{array}$ & $\begin{array}{c}28.99 \pm 1.16 \\
0.793\end{array}$ & $\begin{array}{c}0.0130 \pm 0.0009 \\
0.407\end{array}$ & $\begin{array}{c}0.0278 \pm 0.0064 \\
<0.001\end{array}$ & $\begin{array}{c}0.0368 \pm 0.0002 \\
<0.001\end{array}$ & $\begin{array}{c}4.9948 \pm 0.0191 \\
<0.001\end{array}$ \\
\hline
\end{tabular}

* One-way analysis of variance. + Two-tailed, independent two-sample $t$-test. $\ddagger$ Pixel values (mean \pm standard deviation). CC, contrast medium concentration (\%); $\mathrm{CT}$, computed tomography; $\mathrm{MF}$, manufacturer.

\section{2. $T A$ of $S R$}

There were no statistically significant differences in the SR TA descriptors in the four imaging locations within each CT scanner $(p>0.05)$. However, there were statistically significant differences in all six SR TA descriptors $(p<0.001)$ between appropriate images acquired with the different $\mathrm{CT}$ scanner models within the same manufacturer and distorted images acquired with the different CT scanner models of all manufacturers (Table 4). There were no statistically significant differences in contrast, softness, skewness, uniformity, or randomness index values for appropriate or distorted images between Siemens scanner models. However, regarding the brightness index, values were statistically significantly different for distorted images (Dist_B, $p<0.001$; Dist_F, $p=0.003$ ) (Table 5). For appropriate images acquired using GE CT scanners, the contrast, softness, and skewness index values were more similar than any other values ( $p>0.1$ for all three) (Table 5). There were no differences for any descriptors of distorted images (i.e., Dist_B, and Dist_F) between the GE scanners (all $p>0.05$ ) (Table 5). There were no other significant differences $(p<0.05)$, except in the randomness index for appropriate images acquired by different Phillips CT scanners $(p=0.007)$ and the uniformity index for appropriate images acquired by different Canon CT scanners $(p=0.031)$.

To determine which indices had the highest utility to distinguish between appropriate and distorted images, regardless of the scanner, all SR image data were integrated and compared (Table 4). As the brightness index exhibited statistically significant differences for distorted images between Siemens CT scanners, it was discarded as an unsuitable index. For a combination of the appropriate images, the other five factors achieved a KaiserMeyer-Olkin value of 0.691 which, combined with the result of Bartlett's test of sphericity $(p<0.001)$, indicated that principal component analysis was suitable. Based on the rotated component matrix, the contrast (0.933), softness (0.936), and skewness $(-0.926)$ indices were determined as suitable indices to evaluate the SR. Their suitability is demonstrated via the graphs in Figure 6. As a result, the mean values of contrast, softness, and skewness 
index were $29.09 \pm 0.28,0.0128 \pm 0.0002$, and $-1.6832 \pm 0.0477$, respectively. Here, the skewness index close to 0 represents an image with a symmetrical image.

Table 4. Texture analysis (TA) of appropriate and distorted spatial resolution (SR) images for computed tomography (CT) scanners by different manufacturers.

\begin{tabular}{ccccccc}
\hline MF & Brightness & Contrast & Softness & Skewness & Uniformity & Randomness \\
\hline Siemens $^{*}$ & $235.24 \pm 1.00$ & $29.45 \pm 0.43$ & $0.0132 \pm 0.0004$ & $-1.6360 \pm 0.0705$ & $0.1127 \pm 0.0052$ & $4.2739 \pm 0.0540$ \\
GE $^{*}$ & $241.08 \pm 0.36$ & $28.76 \pm 0.45$ & $0.0126 \pm 0.0004$ & $-1.6606 \pm 0.0765$ & $0.1368 \pm 0.0067$ & $3.8993 \pm 0.0441$ \\
Philips $^{*}$ & $238.79 \pm 0.84$ & $29.08 \pm 0.54$ & $0.0128 \pm 0.0005$ & $-1.6903 \pm 0.0885$ & $0.1046 \pm 0.0038$ & $4.2339 \pm 0.0644$ \\
Canon $^{*}$ & $238.80 \pm 0.86$ & $29.06 \pm 0.47$ & $0.0128 \pm 0.0004$ & $-1.7464 \pm 0.0826$ & $0.0926 \pm 0.0035$ & $4.3578 \pm 0.0447$ \\
Distorted data $^{+}$ & $235.26 \pm 2.09$ & $31.72 \pm 1.36$ & $0.0153 \pm 0.0013$ & $-1.8544 \pm 0.1293$ & $0.0882 \pm 0.0107$ & $4.5971 \pm 0.1361$ \\
-value $^{\ddagger}$ & $<0.001$ & $<0.001$ & $<0.001$ & $<0.001$ & $<0.001$ & $<0.001$ \\
\hline
\end{tabular}

* These are the mean values of appropriate images acquired with the different CT scanner models of the same manufacturer. + These are the mean values of distorted images acquired with the different CT scanner models of all manufacturers. $\ddagger$ Two-tailed, independent two-sample $t$-test. All texture analysis (TA) values are provided as the mean \pm standard deviation. CT, computed tomography; MF, manufacturer.

Table 5. Texture analysis (TA) of appropriate and distorted spatial resolution (SR) images for Siemens and GE computed tomography (CT) scanners.

\begin{tabular}{|c|c|c|c|c|c|c|c|c|}
\hline & MF & Models & Brightness & Contrast & Softness & Skewness & Uniformity & Randomness \\
\hline \multirow{7}{*}{ Ap } & \multirow[b]{3}{*}{ Siemens } & AS & $234.51 \pm 0.61$ & $29.42 \pm 0.27$ & $0.0131 \pm 0.0002$ & $-1.6252 \pm 0.0457$ & $0.1128 \pm 0.0061$ & $4.2796 \pm 0.0548$ \\
\hline & & AS+ & $235.75 \pm 1.27$ & $29.66 \pm 0.49$ & $0.0134 \pm 0.0004$ & $-1.6696 \pm 0.0827$ & $0.1146 \pm 0.0060$ & $4.2565 \pm 0.0677$ \\
\hline & & EDGE & $235.36 \pm 0.62$ & $29.27 \pm 0.44$ & $0.0130 \pm 0.0004$ & $-1.6117 \pm 0.0711$ & $0.1109 \pm 0.0031$ & $4.2863 \pm 0.0396$ \\
\hline & \multirow{5}{*}{ GE } & $p$-value * & 0.069 & 0.250 & 0.246 & 0.293 & 0.429 & 0.583 \\
\hline & & $\mathrm{HD}$ & $241.29 \pm 0.20$ & $28.85 \pm 0.56$ & $0.0126 \pm 0.0005$ & $-1.6725 \pm 0.0973$ & $0.1407 \pm 0.0056$ & $3.8776 \pm 0.0423$ \\
\hline & & VCT & $240.87 \pm 0.37$ & $28.67 \pm 0.32$ & $0.0125 \pm 0.0003$ & $-1.6487 \pm 0.0537$ & $0.1328 \pm 0.0054$ & $3.9210 \pm 0.0365$ \\
\hline & & $p$-value ${ }^{\dagger}$ & 0.024 & 0.464 & 0.453 & 0.584 & 0.019 & 0.062 \\
\hline \multirow{7}{*}{ Dist_B } & & AS & $236.16 \pm 0.57$ & $32.18 \pm 0.61$ & $0.0157 \pm 0.0006$ & $-1.8396 \pm 0.0806$ & $0.0913 \pm 0.0082$ & $4.5993 \pm 0.1256$ \\
\hline & \multirow{3}{*}{ Siemens } & AS+ & $232.50 \pm 1.49$ & $33.00 \pm 1.16$ & $0.0167 \pm 0.0009$ & $-1.9409 \pm 0.1219$ & $0.0873 \pm 0.0038$ & $4.6718 \pm 0.0658$ \\
\hline & & EDGE & $232.78 \pm 0.88$ & $32.54 \pm 0.63$ & $0.0160 \pm 0.0061$ & $-1.9192 \pm 0.0613$ & $0.0901 \pm 0.0028$ & $4.6237 \pm 0.0425$ \\
\hline & & $p$-value * & $<0.001$ & 0.600 & 0.590 & 0.121 & 0.400 & 0.296 \\
\hline & \multirow{4}{*}{ GE } & $\mathrm{HD}$ & $237.81 \pm 0.61$ & $30.97 \pm 0.44$ & $0.0145 \pm 0.0004$ & $-1.8032 \pm 0.0498$ & $0.1048 \pm 0.0078$ & $4.3761 \pm 0.0793$ \\
\hline & & VCT & $237.38 \pm 0.84$ & $31.71 \pm 1.14$ & $0.0152 \pm 0.0011$ & $-1.8998 \pm 0.1301$ & $0.1004 \pm 0.0036$ & $4.4054 \pm 0.04300$ \\
\hline & & $p$-value ${ }^{+}$ & 0.291 & 0.145 & 0.142 & 0.105 & 0.206 & 0.411 \\
\hline \multirow{7}{*}{ Dist_F } & & AS & $234.59 \pm 0.32$ & $33.07 \pm 1.72$ & $0.0166 \pm 0.0017$ & $-1.9463 \pm 0.2338$ & $0.0856 \pm 0.0056$ & $4.7064 \pm 0.0893$ \\
\hline & \multirow{3}{*}{ Siemens } & AS+ & $232.47 \pm 1.61$ & $32.76 \pm 0.83$ & $0.0162 \pm 0.0008$ & $-1.9046 \pm 0.0858$ & $0.0891 \pm 0.0027$ & $4.6590 \pm 0.0494$ \\
\hline & & EDGE & $232.60 \pm 0.92$ & $32.57 \pm 0.76$ & $0.0161 \pm 0.0007$ & $-1.8752 \pm 0.0876$ & $0.0867 \pm 0.0043$ & $4.6740 \pm 0.0685$ \\
\hline & & $p$-value * & 0.003 & 0.735 & 0.710 & 0.686 & 0.326 & 0.459 \\
\hline & \multirow{3}{*}{ GE } & HD & $237.29 \pm 0.68$ & $32.15 \pm 0.43$ & $0.0157 \pm 0.0004$ & $-1.9055 \pm 0.0679$ & $0.0984 \pm 0.0063$ & $4.4629 \pm 0.1029$ \\
\hline & & VCT & $236.53 \pm 0.62$ & $32.10 \pm 0.77$ & $0.0156 \pm 0.0007$ & $-1.9431 \pm 0.0995$ & $0.1012 \pm 0.0053$ & $4.4468 \pm 0.0970$ \\
\hline & & $p$-value ${ }^{\dagger}$ & 0.79 & 0.884 & 0.893 & 0.426 & 0.392 & 0.768 \\
\hline
\end{tabular}

* One-way analysis of variance. + Two-tailed, independent two-sample $t$-test. Ap, appropriate image; CT, computed tomography; Dist_B, distorted image (gantry tilted 10 degrees backward); Dist_F, distorted image (gantry tilted 10 degrees forward); MF, manufacturer.

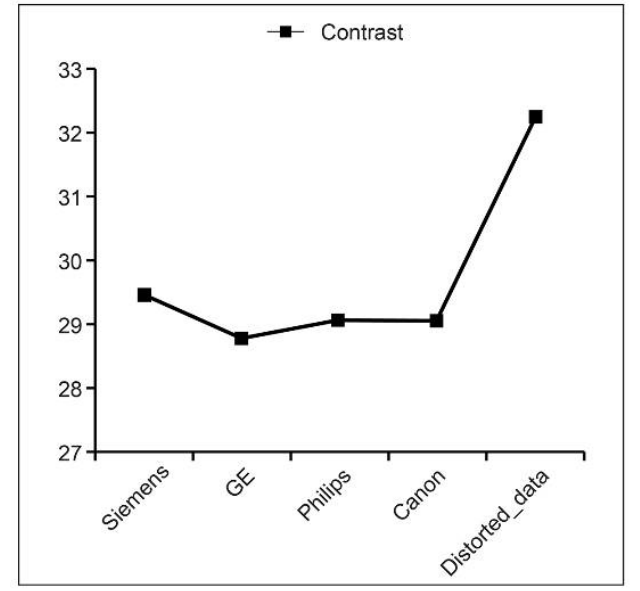

(a)

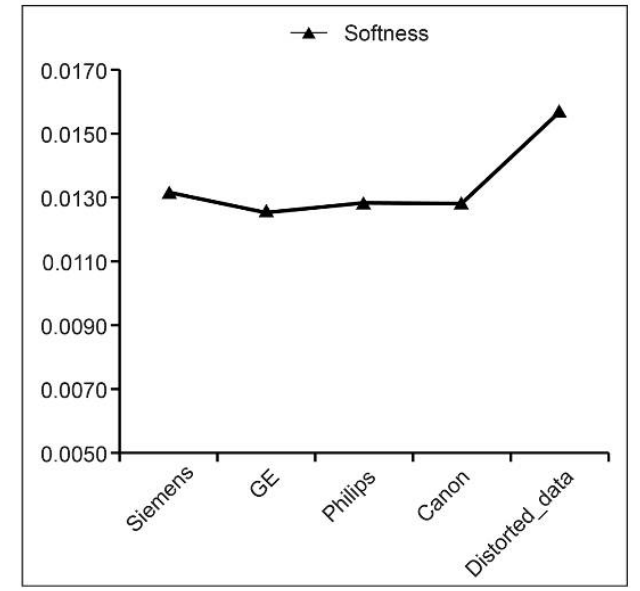

(b)

Figure 6. Cont. 


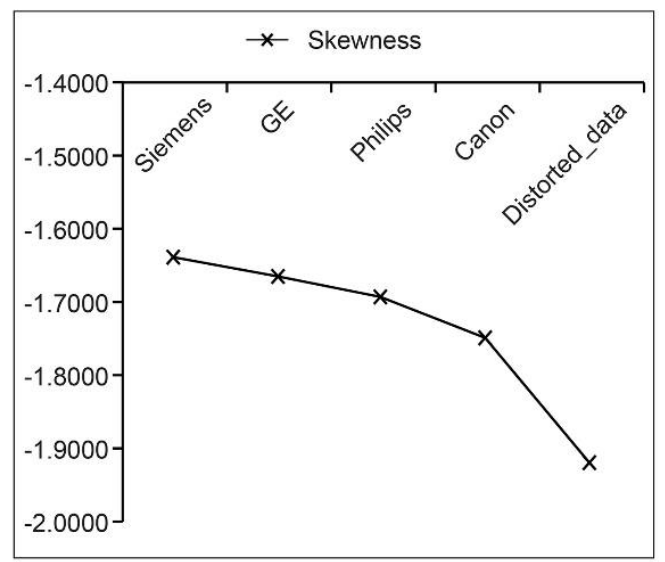

(c)

Figure 6. Textural analysis of appropriate images per manufacturer and all distorted images combined, for spatial resolution (SR). (a) Mean values of the contrast index; (b) mean values of the softness index; (c) mean values of the skewness index.

\section{Discussion}

In QC of CT scanners, CR and SR evaluation with the standard phantom has often been performed subjectively, by eye $[13,14,16]$. Qualitative evaluation in medical diagnostics has an important role to play, but subjective decisions increase the error rate. Different observers, and even the same observer on different occasions, can provide different results when presented with the same signals [35]. In this regard, McCollough et al. [36] revealed common mistakes made by individuals using a phantom when being tested for CT accreditation. Moreover, these analyses should be quantitative in nature, as there is already a subjective component to the assessment of clinical CT images [16,18]. Additionally, the criteria used in the phantom evaluation should be consistent. Currently, in Europe and the United States, it is mainly recommended that CNR should be used for CR evaluation and modulation transfer function (MTF) or image sharpness assessment for SR analysis [16,17]. CNR is also a useful index for distinguishing at low contrast, but it cannot reflect a whole CR image containing all sizes of holes by using a small size of ROI. Moreover, the acceptance criteria for $\mathrm{CR}$ include that circles of certain sizes should be shown (e.g., circles up to $6.4 \mathrm{~mm}$ in diameter must be distinguished from the background with the AAPM phantom [14,18]), and all four cylinders (diameters: 2,3,4,5, and $6 \mathrm{~mm}$ ) are visualized by using the ACR phantom [17,37]. For this reason, it would be better to quantify the entire CR image, as was performed in this study. For MTF measurement for SR analysis, line spread function or edge spread function should be acquired by specific images in the form of straight lines and then transformed into the spatial-frequency domain [38]. Although MTF is a useful method, the direct SR block images of the standard phantom could not be utilized (i.e., a slanted image or a point image with a clear difference in contrast is essential). In contrast, our method is easier to apply and has the advantage that a variety of images, including the AAPM phantom, can be used directly; this can simplify complex processes by only setting the ROI on the SR image.

Depending on the equipment used, the values of CTDIvol were different even if the same tube currents (mAs) were applied in the AAPM phantom (Table 1). It is important to control for radiation dose when quantitatively comparing different $\mathrm{CT}$ scanners because the radiation dose is related to $\mathrm{kVp}$ and $\mathrm{mA}$, which directly affects the signal-to-noise ratio, not the resolution. Therefore, in our study, a dose-based protocol was utilized (Table 1), similar to that used by Saiprasad et al. [28] and Gulliksrud et al. [39].

As a result, the feasible TA descriptors were determined for CR and SR evaluation. Among the TA descriptors, brightness, uniformity, and randomness were inappropriate as useful criteria for CR and SR. Since the value of tube currents was different even at the same CTDIvol among all the scanners, there may have been a statistically significant 
difference in brightness representing the number of X-rays. Uniformity represents a uniform image, whereas randomness represents a non-uniform image. In other words, these descriptors emphasize the characteristics whether they are uniform or not. Therefore, we assumed that these maybe did not fit the TA, the ability to distinguish two different objects. We recommend the following indices and ranges as suitable values: CR: contrast index, $29.5 \pm 15 \%$; softness index, $<0.015$; SR: contrast index, $29 \pm 10 \%$; softness index, $<0.014$; and skewness index, $>-1.85$. The recommended values were slightly expanded beyond the range of the previous results section as a conservative threshold because it may be dangerous to determine appropriate or inadequate values within a tight range setting. Additionally, the range was set in consideration of the radiation intensity and volume. For contrast index, the range of $15 \%$ for $\mathrm{CR}$ and $10 \%$ for SR were set considering our numerical results and regulation of tube current $(\mathrm{mA})$ involved in CTDIvol or tube voltages $(\mathrm{kVp})$. The allowed ranges of $\mathrm{mAs}$ and CTDIvol related to CR are $\pm 15 \%$ and those of $\mathrm{kVp}$ related to $\mathrm{SR}$ are $\pm 10 \%[15,16]$. Based on our results and the regulation of the $\mathrm{QC}$, we presented the appropriate ranges making the standards more flexible.

TA using a histogram is divided into several types such as first-order, and second-order methods $[29,30]$. The first-order method, which is the design of this study, measures texture calculated using only a histogram. The second-order method could take into account the relative position between pixels along with the existing brightness distribution. It is called a gray-level co-occurrence matrix (GLCM), which creates a co-occurrence matrix based on the frequency count of one pixel and another neighboring pixel value in the original image $[29,30]$. However, as a result of using GLCM when evaluating CR and SR using the AAPM phantom, there was no appearance of appropriate values to distinguish subtle differences or changes in phantom images. On the other hand, the first-order method could classify numerically between inappropriate images and normal images. Thus, the firstorder statistical method was adopted since it was possible to find criteria for numerically common convergence in the various pieces of equipment.

There are several limitations to this study. First, we did not perform the analysis with the receiver operating characteristic curve. In future research, the classification of suitable and inappropriate images and $\mathrm{QC}$ with $\mathrm{TA}$ will be performed by collaborating with radiologists. This step will contribute to conducting the exact assessment of the suitability of TA with the receiver operating characteristic curve. Second, the suggested criteria are only applicable to one dose level. Therefore, further studies should be performed at other lower radiation dose levels in order to confirm the maintenance of high quality at low doses. Third, TA analysis was only performed with images of the AAPM phantom. Hence, it will be necessary to evaluate the feasibility of other QC phantoms. Lastly, even if there is no statistically significant difference, there may be limitations in concluding that the proposed parameters are suitable for QC. Therefore, a verification of the statistical significance of the results is proposed through further research in the future. Despite these limitations, this was the first study conducted to present the TA method and the criteria for evaluating $\mathrm{CR}$ and SR for any CT scanner. Although additional verification may be required, our study showed the feasibility of TA for quantifying CR and SR evaluation with the AAPM phantom. Especially the first-order statistical TA method proved to be efficient in the quantification of CT QC.

\section{Conclusions}

Our novel TA method may improve the assessment of CR and SR of AAPM phantom images and standardize the CT QC process instead of subjective visual evaluation. The proposed TA descriptors were contrast, and softness index for CR, and contrast, softness, and skewness index for SR. Therefore, this study presented an opportunity to easily perform QC in hospitals of various sizes by presenting an automated QC method for image-based diagnostic medical equipment. 
Author Contributions: Conceptualization: H.C.K.; methodology: K.B.L. and K.C.N.; validation: K.B.L.; formal analysis: K.B.L. and J.S.J.; investigation: K.B.L.; resources: K.B.L.; data curation: K.C.N.; writing—original draft preparation: K.B.L.; writing—review and editing: K.C.N. and J.S.J.; visualization: K.B.L.; supervision: H.C.K.; funding acquisition: H.C.K. All authors have read and agreed to the published version of the manuscript.

Funding: This research was funded by Eulji University, grant number EJBS-19-04; and the National Research Foundation of Korea (NRF) grant funded by the Korea government (MSIT), grant number 2019R1H1A1079770.

Institutional Review Board Statement: Not applicable.

Informed Consent Statement: Not applicable.

Data Availability Statement: The datasets used and/or analyzed during the current study are available from the corresponding author on reasonable request.

Acknowledgments: We would like to thank Eulji University (www.eulji.ac.kr (accessed on 23 February 2021)).

Conflicts of Interest: The authors declare no conflict of interest. The funders had no role in the design of the study; in the collection, analyses, or interpretation of data; in the writing of the manuscript; or in the decision to publish the results.

\section{References}

1. Zeng, G.L. Medical Image Reconstruction: A Conceptual Tutorial; Springer: Heidelberg, Germany, 2010; pp. 67-85.

2. Jeong, J.E.; Lee, S.J. Performance comparison of ray-driven system models in model-based iterative reconstruction for transmission computed tomography. J. Biomed. Eng. Res. 2014, 35, 142-150. [CrossRef]

3. Amis, E.S., Jr.; Butler, P.F.; Applegate, K.E.; Birnbaum, S.B.; Brateman, L.F.; Hevezi, J.M.; Mettler, F.A.; Morin, R.L.; Pentecost, M.J.; Smith, G.G.; et al. American College of Radiology white paper on radiation dose in medicine. J. Am. Coll. Radiol. 2007, 4, 272-284. [CrossRef]

4. Do, K.H.; Sung, D.W. Strategies of computed tomography radiation dose reduction: Justification and optimization. J. Korean Med. Assoc. 2015, 58, 534-541. [CrossRef]

5. Shefer, E.; Altman, A.; Behling, R.; Goshen, R.; Gregorian, L.; Roterman, Y.; Uman, I.; Wainer, N.; Yagil, Y.; Zarchin, O. State of the art of CT detectors and sources: A literature review. Curr. Radiol. Rep. 2013, 1, 76-91. [CrossRef]

6. Geyer, L.L.; Glenn, G.R.; De Cecco, C.N.; Van Horn, M.; Canstein, C.; Silverman, J.R.; Krazinski, A.W.; Kemper, J.M.; Bucher, A.; Ebersberger, U.; et al. CT Evaluation of small-diameter coronary artery stents: Effect of an integrated circuit detector with iterative reconstruction. Radiology 2015, 276, 706-714. [CrossRef] [PubMed]

7. Zhu, Z.; Zhao, X.M.; Zhao, Y.F.; Wang, X.Y.; Zhou, C.W. Feasibility study of using Gemstone Spectral Imaging (GSI) and adaptive statistical iterative reconstruction (ASIR) for reducing radiation and iodine contrast dose in abdominal CT patients with high BMI values. PLOS ONE 2015, 10, e0129201. [CrossRef]

8. Kalra, M.K.; Maher, M.M.; Toth, T.L.; Hamberg, L.M.; Blake, M.A.; Shepard, J.A.; Saini, S. Strategies for CT radiation dose optimization. Radiology 2004, 230, 619-628. [CrossRef] [PubMed]

9. Lee, K.; Lee, W.; Lee, J.; Lee, B.; Oh, G. Dose reduction and image quality assessment in MDCT using AEC (D-DOM \& Z-DOM) and in-plane bismuth shielding. Radiat. Prot. Dosimetry 2010, 141, 162-167. [CrossRef] [PubMed]

10. Gunn, M.L.D.; Kohr, J.R. State of the art: Technologies for computed tomography dose reduction. Emerg Radiol. 2010, 17, 209-218. [CrossRef]

11. Brenner, D.J.; Hall, E.J. Computed tomography-An increasing source of radiation exposure. N. Engl. J. Med. 2007, 357, 2277-2284. [CrossRef] [PubMed]

12. Larson, D.B.; Johnson, L.W.; Schnell, B.M.; Salisbury, S.R.; Forman, H.P. National trends in CT use in the emergency department: 1995-2007. Radiology 2011, 258, 164-173. [CrossRef]

13. ICRP; Rehani, M.M.; Gupta, R.; Bartling, S.; Sharp, G.C.; Pauwels, R.; Berris, T.; Boon, J.M. Radiological protection in cone beam computed tomography (CBCT). ICRP publication 129. Ann. ICRP 2015, 44, 9-127. [CrossRef]

14. Park, H.J.; Jung, S.E.; Lee, Y.J.; Cho, W.I.; Do, K.H.; Kim, S.H.; Na, D.G. Review of failed CT phantom image evaluations in 2005 and 2006 by the CT accreditation program of the korean institute for accreditation of medical image. Korean J. Radiol. 2008, 9 , 354-363. [CrossRef]

15. New Jersey Department of Environmental Protection. Compliance Guidance for Computed Tomography Quality Control, 2nd ed.; Bureau of X-ray Compliance: Trenton, NJ, USA. Available online: https://www.state.nj.us/dep/rpp/qa/qa_down/qaman.pdf (accessed on 19 December 2020).

16. European Commission. Radiation protection $\mathrm{N}^{\circ}$ 162: Criteria for Acceptability of Medical Radiological Equipment Used in Diagnostic Radiology, Nuclear Medicine and Radiotherapy. Quality Assurance Reference Centre for the European Commission 2012. Available online: https:/ / ec.europa.eu/energy/sites/ener/files/documents/162.pdf (accessed on 28 March 2021). 
17. ACR Committee on CT Accreditation, 2017 Computed Tomography Quality Control Manual; American College of Radiology: Reston, VA, USA; Available online: https://www.acr.org/-/media/ACR/NOINDEX/QC-Manuals/CT_QCManual.pdf/ (accessed on 23 February 2021).

18. Lee, K.B.; Cho, Y.B.; Jeong, H.K.; Nam, K.C.; Kim, H.C. The study on automatized quantitative assessment method of CT Image in quality control: Focusing on spatial and low contrast resolution. J. IEIE 2017, 54, 186-194. [CrossRef]

19. Sharp, P.; Barber, D.C.; Brown, D.G.; Burgess, A.E.; Metz, C.E.; Myers, K.J.; Taylor, C.J.; Wagner, R.F.; Brooks, R.; Hill, C.R.; et al. ICRU Report 54. Medical imaging—the assessment of image quality. Rep. Int. Comm. Radiat. Units Meas. 1996, os28, 1-41. [CrossRef]

20. Lee, K.B.; Goo, H.W. Quantitative image quality and histogram-based evaluations of an iterative reconstruction algorithm at low-to-ultralow radiation dose levels: A phantom study in chest CT. Korean J. Radiol. 2018, 19, 119-129. [CrossRef]

21. Hodgdon, T.; McInnes, M.D.F.; Schieda, N.; Flood, T.A.; Lamb, L.; Thornhill, R.E. Can quantitative CT texture analysis be used to differentiate fat-poor renal angiomyolipoma from renal cell carcinoma on unenhanced CT images? Radiology 2015, 276, 787-796. [CrossRef] [PubMed]

22. Wang, X.; Yuan, M.; Mi, H.; Suo, S.; Eteer, K.; Li, S.; Lu, Q.; Xu, J.; Hu, J. The feasibility of differentiating colorectal cancer from normal and inflammatory thickening colon wall using CT texture analysis. Sci. Rep. 2020, 10, 6346. [CrossRef] [PubMed]

23. Ganeshan, B.; Miles, K.A. Quantifying tumour heterogeneity with CT. Cancer Imaging 2013, 13, 140-149. [CrossRef] [PubMed]

24. Andersen, M.B.; Harders, S.W.; Ganeshan, B.; Thygesen, J.; Torp Madsen, H.H.; Rasmussen, F. CT texture analysis can help differentiate between malignant and benign lymph nodes in the mediastinum in patients suspected for lung cancer. Acta Radiol. 2016, 57, 669-676. [CrossRef]

25. Bharati, M.H.; Liu, J.J.; MacGregor, J.F. Image texture analysis: Methods and comparisons. Chemom. Intell. Lab. Syst. 2004, 72, 57-71. [CrossRef]

26. Di Cataldo, S.; Ficarra, E. Mining textural knowledge in biological images: Applications, methods and trends. Comput. Struct. Biotechnol. J. 2016, 15, 56-67. [CrossRef] [PubMed]

27. Miles, K.A.; Ganeshan, B.; Hayball, M.P. CT texture analysis using the filtration-histogram method: What do the measurements mean? Cancer Imaging 2013, 13, 400-406. [CrossRef] [PubMed]

28. Saiprasad, G.; Filliben, J.; Peskin, A.; Siegel, E.; Chen, J.; Trimble, C.; Yang, Z.; Christianson, O.; Samei, E.; Krupinski, E.; et al. Evaluation of low-contrast detectability of iterative reconstruction across multiple institutions, CT scanner manufacturers, and radiation exposure levels. Radiology 2015, 277, 124-133. [CrossRef] [PubMed]

29. Srinivasan, G.N.; Shobha, G. Statistical texture analysis. Proc. World Acad. Sci. Eng. Technol. 2008, 36, $2070-3740$.

30. Bevk, M.; Kononenko, I. A Statistical approach to texture description of medical images: A preliminary study. In Proceedings of the 15th IEEE Symposium Computer-Based Medical Systems (CBMS 2002), Maribor, Slovenia, 4-7 June 2002; pp. 239-244. [CrossRef]

31. Gonzalez, R.C.; Woods, R.E.; Eddins, S.L. Digital Image Processing Using MATLAB, 2nd ed.; Gatesmark Publishing: Knoxville, TN, USA, 2009; pp. 535-655.

32. Abbasian Ardakani, A.A.; Gharbali, A.; Mohammadi, A. Application of texture analysis method for classification of benign and malignant thyroid nodules in ultrasound images. Iran. J. Cancer Prev. 2015, 8, 116-124. [CrossRef]

33. Lee, J.; Im, I.; Yu, Y.; Park, H.; Kwak, B. Statistical techniques based computer-aided diagnosis (CAD) using texture feature analysis: Applied of cerebral infarction in computed tomography (CT) images. Biomed. Sci. Lett. 2012, 18, 399-405.

34. Principal Components Analysis (PCA) Using SPSS Statistics. Available online: https://statistics.laerd.com/spss-tutorials/ principal-components-analysis-pca-using-spss-statistics.php/ (accessed on 21 December 2020).

35. Thilander-Klang, A.; Ledenius, K.; Hansson, J.; Sund, P.; Båth, M. Evaluation of subjective assessment of the low-contrast visibility in constancy control of computed tomography. Radiat. Prot. Dosim. 2010, 139, 449-454. [CrossRef]

36. McCollough, C.H.; Bruesewitz, M.R.; McNitt-Gray, M.F.; Bush, K.; Ruckdeschel, T.; Payne, J.T.; Brink, J.A.; Zeman, R.K.; American College of Radiology. The phantom portion of the American College of Radiology (ACR) Computed Tomography (CT) accreditation program: Practical tips, artifact examples, and pitfalls to avoid. Med. Phys. 2004, 31, 2423-2442. [CrossRef]

37. American College of Radiology. Computed Tomography Accreditation Program Phantom Testing Instructions. Available online: http:/ / www.doza.ru/docs/med/phantom_testing_instruction.pdf (accessed on 20 December 2020).

38. Boone, J.M.; Brink, J.A.; Huda, W.; Leitz, W.; McCollough, C.H.; McNitt-Gray, M.F.; Dawson, P.; Deluca, P.L.M.; Seltzer, S.M.; Brunberg, J.A.; et al. Radiation dose and image-quality assessment in computed tomography. J. ICRU 2012, 12, 9-149. [CrossRef]

39. Gulliksrud, K.; Stokke, C.; Martinsen, A.C.T. How to measure CT image quality: Variations in CT-numbers, uniformity and low contrast resolution for a CT quality assurance phantom. Phys. Med. 2014, 30, 521-526. [CrossRef] [PubMed] 